\title{
28 Research Suare \\ Nonalcoholic fatty liver disease as a risk factor for severity of acute cholangitis
}

Mahamid Mahmud

Shaare Zedek Medical Center

Bashar Fteiha ( $\sim$ basharf@szmc.org.il )

Shaare Zedek Medical Center https://orcid.org/0000-0001-7676-9447

Nubani Adi

Shaare Zedek Medical Center

Eran Goldin

Shaare Zedek Medical Center

Wiliam Nseir

Baruch Padeh Medical Center Poriya

\section{Research article}

Keywords: Nonalcoholic fatty liver disease, Cholangitis, Metabolic syndrome, Gut-liver axis

Posted Date: August 27th, 2020

DOI: https://doi.org/10.21203/rs.3.rs-56038/v1

License: (1) This work is licensed under a Creative Commons Attribution 4.0 International License.

Read Full License 


\section{Abstract}

Background: Nonalcoholic fatty liver disease (NAFLD) is one of the most prevalent chronic liver disorders worldwide. Acute cholangitis (AC) is a serious and life-threatening illness. We aimed to determine whether NAFLD is a risk factor for severity of AC.

Methods: We retrospectively studied hospitalized patients with diagnosis of AC over the span of 5 years. Subjects were divided into a NAFLD group and Non NAFLD group. We compared between the two groups in terms of demographic characteristics, comorbidities, laboratory data, and severity of AC (including Charlson Comorbidity index and Tokyo Consensus meeting criteria).

Results: 298 of 419 patients who had AC and were hospitalized met the inclusion criteria and were included in the study. Of them, 73/298 (24.5\%) were included in NAFLD group. NAFLD group patients were younger, diabetic and more obese than non NAFLD group. Subjects in the NAFLD presented with higher serum C-reactive protein and higher liver enzymes ( $p<0.05$, for each parameter) and suffered more events of organ dysfunction $(p<0.001)$ and bacteremia $(p<0.005)$. Regarding the severity of AC according to Tokyo classification, among the NAFLD group more subjects presented with Grade II (39.7 Vs $33.3 \%$, $p$ $<0.001)$ and Grade III (23.3 vs 18.3, $\mathrm{p}<0.001)$ cholangitis, as opposed more Grade I cholangitis among the non-NAFLD group ( 48.4 vs $37 \%, p<0.001$ ). Multivariate logistic regression analysis showed that NAFLD is independently associated with severe AC, grade III (OR 3.25, 95\% Cl 1.65-6.45, p=0.038).

Conclusions: NAFLD is as an independent risk factor for severity of AC.

\section{Background}

Nonalcoholic fatty liver disease (NAFLD) is one of the most prevalent chronic liver disorders worldwide [1]. This entity encompasses a wide spectrum of hepatic damage in which steatosis with inflammation progresses to non-alcoholic steatohepatitis (NASH), fibrosis, cirrhosis, and, ultimately, hepatocellular carcinoma[1, 2]. Biliary and pancreatic manifestations have been reported in patients with NAFLD. NAFLD has been positively found to be associated with pancreatitis and its severity [3-8].

NAFLD is associated with metabolic syndrome and is considered the hepatic manifestation of the metabolic syndrome. Moreover, NAFLD is linked with several components of the metabolic syndrome, primarily diabetes mellitus and obesity $[9,10]$. Importantly, a pathogenetic link between NAFLD and imbalance of the gut microbiome has been observed. Recent studies have indicated that changes in gut microbiome is associated with NAFLD and progression to NASH [11, 12].

Acute cholangitis (AC) is a clinical syndrome characterized by fever, jaundice, and abdominal pain that develops as a result of stasis and infection in the biliary tract. It is also referred to as ascending cholangitis. Cholangitis was first described by Charcot as a serious and life-threatening illness; however, it is now recognized that the severity can range from mild to life-threatening [13] . 
As in NAFLD gut microbiome play a crucial role in the pathogenesis of acute cholangitis via an interesting recently described Gut-Liver Axis and Inflammasome Activation in Cholangiocyte Pathophysiology [14] .

There are no data on the incidence, clinical course and outcomes of Cholangitis in this fast-growing NAFLD population. The question remains, how does gut microbiome change in NAFLD impact cholangitis susceptibility. The aim of this study is to determine whether NAFLD is a risk factor for severity of $\mathrm{AC}$.

\section{Methods}

\section{Study population, data collection and protocol design}

This was a retrospective cohort study conducted at the Sharee Zedek Medical Center (SZMC) University Hospital located in Jerusalem, Israel. We reviewed the hospital records of all consecutive adult patients hospitalized over a five-year period (January 2015 to December 2019) at SZMC. Included were adult patients ( $\geq 18$ years) hospitalized for acute cholangitis (The clinical diagnosis of acute cholangitis was made on the basis of the clinical findings, such as Charcot's triad, in combination with the laboratory data and imaging findings) in subjects who underwent an abdominal ultrasonography (US) examination during hospitalization. Patients diagnosed with gastrointestinal infection other than Cholangitis were excluded. Furthermore, patients with known history of heavy alcohol use (defined as subjects who had a history of alcohol consumption of more $>30$ gram a day for at least 5 years) and those diagnosed with chronic viral hepatitis or patients with history of other known liver disease were excluded. Subjects with other hepatic pathology or autoimmune phenotypes (such as alcoholic liver disease, drug-induced liver injury, autoimmune hepatitis, viral hepatitis, cholestatic liver disease and metabolic/genetic liver disease) were also excluded using specific clinical, laboratory, radiological and/or histological criteria/tests. The current study received ethical approval from the local hospital ethical committee and was conducted according to the Helsinki declaration and its subsequent amendments. Data were coded in order to preserve the anonymity of the patients. Informed consent was waived because of the non-interventional study design.

\section{Definitions}

NAFLD was diagnosed by abdominal ultrasonography based on the presence of fatty liver (hepatic parenchymal brightness, visualization of portal and hepatic borders, liver-to-kidney contrast, deep beam attenuation, and bright vessel walls) [15] .

Charlson Comorbidity Index (CCI) predicts 10-year survival/mortality of patients with several comorbidities based on $\mathrm{CCl}$ scoring system. The $\mathrm{CCl}$ score is composed from 17 categories including (age, myocardial infarction, congestive heart failure, peripheral vascular disease, stroke or transient ischemic ischemia, dementia, chronic obstructive pulmonary disease, connective tissue disease, peptic ulcer disease, liver disease, diabetes, hemiplegia, chronic kidney disease, solid tumors, leukemia, lymphoma, and AIDS)[16] . 
Acute cholangitis was defined according to the new diagnostic criteria and severity assessment of acute cholangitis in revised Tokyo Guidelines as: A. Systemic inflammation A-1: Fever and/or shaking chills; A2: Laboratory data: evidence of inflammatory response. B. Cholestasis B-1: Jaundice B-2: abnormal liver function tests. C. Imaging C-1: Biliary dilatation C-2: Evidence of the etiology on imaging (stricture, stone, stent etc.). Suspected diagnosis: one item in A + one item in either B or C Definite diagnosis: one item in A, one item in B and one item in C. Tokyo Consensus Meeting defines severity of AC in 3 grades: grade I, mild, with no onset of organ dysfunction and with a good response to initial antibiotic treatment. Grade II, moderate, with no onset of organ dysfunction but without response to initial antibiotic treatment, and grade III, severe defines as with onset of organ dysfunction and without response to initial antibiotic treatment $[17,18]$.

\section{Statistical analysis}

Before commencing any statistical processing and analysis, data were visually inspected and checked for outliers. The analysis was performed using the commercial software Statistical Package for Social Science (SPSS version 24.0, IBM, Chicago, IL, USA). Categorial variables were tested using the chi-square test or Fisher's exact test, as appropriate. Continuous variables were examined using the student's $t$ test if normally distributed and Man-Whitney test if not. To identify variables associated with the severity of AC, univariate analysis was performed. Variables that were significantly associated $(p<0.1)$ with the primary outcome (severe cases of $\mathrm{AC}$ ) were entered into the multivariate logistic regression model. Adjusted odds-ratios (ORs) and 95\% confidence intervals (Cls) for the severity of AC were calculated. Statistical significance was set at p-value less than 0.05 .

\section{Results}

Of the 419 patients aged $\geq 18$ years hospitalized in the studied period, a total of 121 patients were excluded: 97 did not meet inclusion criteria (indefinite diagnosis of cholangitis or presence of other gastrointestinal active infection on or inflammation) and 24 patients had exclusion criteria (significant alcohol intake in 7, chronic viral hepatitis in 6, cirrhosis in 2 and hepatotoxic medications in 9 patients). Consequently, 298 patients were included in the study. Based upon the results of abdominal ultrasound, the patients were divided into two groups: Subjects with NAFLD $(73,24.5 \%)$ and subjects without NAFLD $(225,75.5 \%)$.

The demographic, clinical, and laboratory data are shown in Table 1. The NAFLD group were younger and predominant of male gender $(53.4 \%$ vs $50.2 \%, p=0.07)$ with a median age of $71.8 \pm 20.9$ years versus $73.6 \pm 19.3$ years in the non-NAFLD group $(p=0.035)$. Obesity and Diabetes mellitus were more frequent among NAFLD group (the mean BMI was $29.7 \pm 7.1$ vs. $26.3 \pm 6.3, p=0.020$; and 29 (39.7\%) versus 74 (32.8\%), $p=0.049$ respectively). There were no significant differences in other comorbidities between the two study groups. 
Table 1

Demographics, clinical and laboratory characteristics of 298 subjects included in each of the study groups

\begin{tabular}{|llll|}
\hline Parameters & NAFLD group & Non-NAFLD group & $\mathrm{P}$ \\
& $\mathrm{n}=73$ & $\mathrm{n}=\mathbf{2 2 5}$ & \\
\hline Age (years), mean \pm SD & $71.8 \pm 20.9$ & $73.6 \pm 19.3$ & 0.034 \\
\hline Male gender, $\mathrm{n}(\%)$ & $39(53.4)$ & $113(50.2)$ & 0.076 \\
\hline Body mass index, mean \pm SD & $29.7 \pm 7.1$ & $26.3 \pm 6.3$ & 0.020 \\
\hline Ischemic heart disease, $\mathrm{n}(\%)$ & $12(16.4)$ & $39(17.3)$ & 0.782 \\
\hline Diabetes mellitus, $\mathrm{n}(\%)$ & $29(39.7)$ & $74(32.8)$ & 0.049 \\
\hline Chronic renal failure, $\mathrm{n}(\%)$ & $14(19.1)$ & $39(17.3)$ & 0.843 \\
\hline Smoking, $\mathrm{n}(\%)$ & $13(17.8)$ & $42(18.6)$ & 0.616 \\
\hline CRP mg/dl, mean $\pm \mathrm{SD}$ & $37.8 \pm 18.6$ & $19.6 \pm 12.2$ & 0.019 \\
\hline Creatinine mg/dl, mean $\pm \mathrm{SD}$ & $1.57 \pm 1.1$ & $1.21 \pm 0.8$ & 0.037 \\
\hline AST U/L, mean \pm SD & $74 \pm 12.8$ & $64.23 \pm 9.43$ & $<0.005$ \\
\hline ALT U/L, mean \pm SD & $61.43 \pm 9.36$ & $49.23 \pm 8.24$ & $<0.005$ \\
\hline GGT U/L, mean \pm SD & $252.3 \pm 73.3$ & $143.25 \pm 91.42$ & $<0.005$ \\
\hline Direct bilirubin mg/dl, mean \pm SD & $6.35 \pm 2.56$ & $2.63 \pm 3.15$ & $<0.001$ \\
\hline Albumin g/dl, mean \pm SD & $2.6 \pm 1.33$ & $3.2 \pm 1.21$ & $<0.005$ \\
\hline Charlson Comorbidity index $>4, \mathrm{n}(\%)$ & $38(16.8)$ & $21(28.7)$ & $<0.005$ \\
\hline Other at least one organ dysfunction, $\mathrm{n}(\%)$ & $19(26.0)$ & $23(10.2)$ & $<0.001$ \\
\hline Bacteremia, $\mathrm{n}(\%)$ & $26(11.1)$ & $17(23.2)$ & $<0.005$ \\
\hline Tokyo classification, $\mathrm{n}(\%)$ & $27(37)$ & $109(48.4)$ & $<0.001$ \\
\hline Grade I & $29(39.7)$ & $75(33.3)$ & $<0.001$ \\
\hline Grade II & $17(23.3)$ & $41(18.3)$ & $<0.001$ \\
\hline Grade III & & & \\
\hline & & & \\
\hline
\end{tabular}

Regarding the severity of AC according to Tokyo classification, among the NAFLD group 27 subjects (37\%) presented with grade I compared to $109(48.4 \%)$ in the non-NAFLD group $(p<0.005), 29(39.7 \%)$ presented with grade II compared to $75(33.3 \%)$ in non-NAFLD subjects $(p<0.005)$, and 17 NAFLD subjects $(23.3 \%)$ presented with grade III cholangitis, compared to $41(18.3 \%)$ in the non-NAFLD group ( $p$ $<0.005)$. The association between NAFLD and the severity of cholangitis is illustrated in Fig. 1 below. 
With regards to laboratory features, NAFLD group subjects had higher serum CRP levels compared to the non-NAFLD group ( $37.8 \mathrm{mg} / \mathrm{dl}$ versus $19.6 \mathrm{mg} / \mathrm{dl}, \mathrm{p}=0.019)$. NAFLD subjects also had higher serum creatinine levels $(1.57 \pm 1.1$ versus $1.21 \pm 0.8 \mathrm{mg} / \mathrm{dl}, p=0.037)$, higher mean AST levels $(74.2 \pm 12.8$ versus 64.23 $\pm 9, p<0.005$ ), mean ALT levels (from $61.43 \pm 9.36$ compared to $49.23 \pm 8.24, p<0.005$ ), mean GGT levels $(252.3 \pm 73.8$ among the NAFLD group versus $143.25 \pm 91.42, \mathrm{P}<0.005)$. Furthermore, a similar trend with direct bilirubin levels as the mean direct bilirubin levels at admission were $6.35 \pm 2.56$ among the NAFLD group versus $2.63 \pm 3.15$ among the non-NAFLD group subjects $(P<0.001)$.

Finally, subjects in the NAFLD group had lower mean serum albumin levels $(2.6 \pm 1.33$ vs, $3.2 \pm 1.21, p<$ 0.005 , respectively), higher frequency of $\mathrm{CCl}>4$ points $(28.7 \%$ vs. $16.8 \%, \mathrm{p}<0.005)$, more events of at least one organ dysfunction (19/73 (26\%) vs. $23 / 225(10.2 \%), p<0.001)$ and more frequent bacteremia $(23 \%$ vs. $11.1 \%, \mathrm{p}<0.005)$ when compared to the non-NAFLD group.

Overall, 28 (9.3\%) patients developed biliary pancreatitis, the majority of which were subjects among the NAFLD group $19(67.85 \%)$ versus $9(32.14 \%)$ among the control group ( $p$ value $<0.001)$. Overall, successful Endoscopic decompression by ERCP was performed in $252(84.56 \%)$ patients and failed ERCP due to technical difficulties was observed in $12(4.02 \%)$ patients. ERCP was waived in $34(11.40 \%)$ subjects due to sepsis and other serious conditions and Percutaneous trans hepatic cholangiography (PTC) was performed for decompression instead. The majority of patients who underwent PTC due to hemodynamic instability or and serious illness were from the NAFLD group (29 (85.3\%) versus 5 (14.7\%), $p<0.001)$.

To identify potential risk factors for severity of $A C$, multivariate logistic regression analysis was performed (Table 2). After adjustment for potential confounders, the Charlson Comorbidity index $>(\mathrm{OR}$ $3.85,95 \% \mathrm{Cl} 1.42-6.5)$, GGT > 60 (OR 3.85, 95\% Cl 2.12-7.54) serum albumin < 2.5 (OR 2.35, 95\% Cl 1.187.36), NAFLD (OR $3.25,95 \% \mathrm{Cl} 1.65-6.45), \mathrm{BMI}>30$ (OR 4.47, 95\% $\mathrm{Cl} 2.53-7.15)$ were all independently associated with severe cholangitis (Grade III). Interestingly, diabetes mellitus was not associated with cholangitis in our model.

Table 2

Multivariate logistic regression analysis to identify potential risk factors for severe AC of grade III

\begin{tabular}{|llll|}
\hline variable & OR & Cl & P value \\
\hline Charlson Comorbidity Index $>4$ & 3.85 & $1.42-6.5$ & 0.015 \\
\hline GGT $>60$ & 3.85 & $2.12-7.54$ & 0.039 \\
\hline serum albumin $<2.5$ & 2.35 & $1.18-7.36$ & 0.042 \\
\hline NAFLD & 3.25 & $1.65-6.45$ & 0.038 \\
\hline BMI $>30 \mathrm{~kg} / \mathrm{m}^{2}$ & 4.47 & $2.53-7.15$ & 0.006 \\
\hline & & & \\
\hline
\end{tabular}




\section{Discussion}

Our study results indicate that NAFLD is strongly associated with severe cholangitis (defined as Tokyo score Grade III). To the best of our knowledge, no previous studies have studied the relationship between NAFLD and severe cholangitis, although a wide array of pancreatobiliary manifestations are commonly found among patients with NAFLD [19] .

Several Recent studies have shown the association between NAFLD and bacterial infections [20-23]. Although the exact mechanism is yet to be revealed, alteration of the immune system function with involvement of the dynamic gut-liver axis seems like the most plausible explanation, presumably through the NLRP3 inflammasome activation and its recently described role in Cholangiocyte pathophysiology [14]. Other possible contributors within this recently described axis are the gut barrier dysfunction and intestinal immune defects that may overburden the liver's defense mechanisms, allowing bacteria to freely enter the circulation [24]. Indeed, more cases of Bacteremia and organ dysfunction were documented among the NAFLD group when compared to the non-NAFLD group in our study.

The mechanisms by which NAFLD may induce severe cholangitis have not been elucidated so far. An increasing body of evidence points towards an increased level of proinflammatory cytokines in patients with NAFLD and non-alcoholic steatohepatitis, with higher oxidative stress and abnormal lipoprotein metabolism also implicated [25]. Furthermore, NAFLD is closely associated with metabolic syndrome and obesity, which is now seen as a chronic low-grade inflammatory state as the adipocytes have been shown to secrete a variety of cytokines like IL-6 and TNF-alpha which promote inflammation [26]. Interestingly, in our study, NAFLD have been shown to be independently related to the occurence of severe cholangitis, regardless of obesity. NAFLD have also been shown to independently correlate with the risk of developing severe pancreatitis, presumably through similar mechanisms [3].

In our study, subjects in the NAFLD group presented with significantly higher CRP levels. This finding is consistent with the results reported from earlier studies where a positive correlation been CRP and NAFLD have been demonstrated [27]. Furthermore, subjects in the NAFLD group had higher Charlson comorbidity index scores, lower albumin levels and higher GGT levels which are all predictors of short-term and longterm mortality $[16,28,29]$. NAFLD has been well established as a predictor of increased all-cause mortality in a recently conducted comprehensive meta-analysis [30]. Similarly, Nseir et al demonstrated an increase in the all-cause 30-day mortality among NAFLD subjects who presented with Communityacquired pneumonia [21].

Our study has several limitations, including the inherent limitations of a retrospective design, a single center study, and its relatively small sample size. Another main limitation is the absence of liver biopsies for the diagnosis of NAFLD. Thus, we didn't correlate with the degree of inflammation in NAFLD subjects and no distinction was made between hepatic steatosis or NASH. On the other hand, this is the first study to describe the association between NAFLD and severe cholangitis as we are not aware of any similar studies published elsewhere in the literature. 


\section{Conclusion}

In conclusion, patients with NAFLD have been shown to be at an increased risk for developing severe cholangitis with higher incidence of bacteremia and organ dysfunction. Given the high prevalence of NAFLD and the substantial morbidity and mortality attributed to severe cholangitis, more rigorous studies are urgently needed to further characterize this important association and effectively reduce morbidity and mortality in this susceptible patient population.

\section{Abbreviations}

NAFLD, nonalcoholic fatty liver disease. AC, Acute cholangitis. NASH, non-alcoholic steatohepatitis. CCl, Charlson comorbidity index. AIDS, acquired immune deficiency syndrome. ERCP, endoscopic retrograde cholangio-pancreatography.

\section{Declarations}

Ethics approval and consent to participate - The current study received ethical approval from the Helsinki Committee of Shaare Zedek Medical Center (reference number SZMC-19-0379) and was conducted according to the Helsinki declaration and its subsequent amendments

Consent for publication - Not applicable

Availability of data and materials - The datasets used and/or analyzed during the current study are available from the corresponding author on reasonable request.

Competing interests - The authors declare that they have no competing interests

Funding - No funding was received by the authors for the completion of this work

Acknowledgments - Not applicable.

\section{Authors' contributions:}

$\mathrm{MM}$ - designed and conducted the study, collected data and proofed the manuscript.

BF - designed and conducted the study, collected data and proofed the manuscript.

AN - collected data and proofed the manuscript.

EG - proofed the manuscript.

WN - reviewed the manuscript, performed the statistical analysis.

all authors have read and approved the manuscript. 


\section{References}

[1] Fassio E, Alvarez E, Domínguez N, Landeira G, Longo C. Natural history of nonalcoholic steatohepatitis: a longitudinal study of repeat liver biopsies. Hepatology. 2004;40:820-6. https://doi.org/10.1002/hep.20410.

[2] Ahmed A, Wong RJ, Harrison SA. Nonalcoholic Fatty Liver Disease Review: Diagnosis, Treatment, and Outcomes. Clin Gastroenterol Hepatol Off Clin Pract J Am Gastroenterol Assoc. 2015;13:2062-70. https://doi.org/10.1016/j.cgh.2015.07.029.

[3] Hou S, Tang X, Cui H, Liu C, Bai X, Shi L, et al. Fatty liver disease is associated with the severity of acute pancreatitis:A systematic review and meta-analysis. Int J Surg. 2019;65:147-53. https://doi.org/10.1016/j.ijsu.2019.04.003.

[4] Mikolasevic I, Orlic L, Poropat G, Jakopcic I, Stimac D, Klanac A, et al. Nonalcoholic fatty liver and the severity of acute pancreatitis. Eur J Intern Med. 2017;38:73-8. https://doi.org/10.1016/j.ejim.2016.10.019.

[5] Uygun A, Kadayifci A, Demirci H, Saglam M, Sakin YS, Ozturk K, et al. The effect of fatty pancreas on serum glucose parameters in patients with nonalcoholic steatohepatitis. Eur $\mathrm{J}$ Intern Med. 2015;26:37-41. https://doi.org/10.1016/j.ejim.2014.11.007.

[6] Idilman IS, Tuzun A, Savas B, Elhan AH, Celik A, Idilman R, et al. Quantification of liver, pancreas, kidney, and vertebral body MRI-PDFF in non-alcoholic fatty liver disease. Abdom Imaging. 2015;40:1512-9. https://doi.org/10.1007/s00261-015-0385-0.

[7] Koller T, Kollerova J, Hlavaty T, Huorka M, Payer J. Cholelithiasis and markers of nonalcoholic fatty liver disease in patients with metabolic risk factors. Scand J Gastroenterol. 2012;47:197-203. https://doi.org/10.3109/00365521.2011.643481.

[8] Loria P, Lonardo A, Lombardini S, Carulli L, Verrone A, Ganazzi D, et al. Gallstone disease in nonalcoholic fatty liver: prevalence and associated factors. J Gastroenterol Hepatol. 2005;20:1176-84. https://doi.org/10.1111/j.1440-1746.2005.03924.x.

[9] Younossi ZM, Koenig AB, Abdelatif D, Fazel Y, Henry L, Wymer M. Global epidemiology of nonalcoholic fatty liver disease-Meta-analytic assessment of prevalence, incidence, and outcomes. Hepatology. 2016;64:73-84. https://doi.org/10.1002/hep.28431.

[10] Clark JM, Diehl AM. Defining nonalcoholic fatty liver disease: implications for epidemiologic studies. Gastroenterology. 2003;124:248-50. https://doi.org/10.1053/gast.2003.50032.

[11] Iruzubieta P, Medina JM, Fernández-López R, Crespo J, de la Cruz F. A Role for Gut Microbiome Fermentative Pathways in Fatty Liver Disease Progression. J Clin Med. 2020;9. https://doi.org/10.3390/jcm9051369. 
[12] Ji Y, Yin Y, Sun L, Zhang W. The Molecular and Mechanistic Insights Based on Gut-Liver Axis: Nutritional Target for Non-Alcoholic Fatty Liver Disease (NAFLD) Improvement. Int J Mol Sci. 2020;21:3066. https://doi.org/10.3390/ijms21093066.

[13] Sulzer JK, Ocuin LM. Cholangitis: Causes, Diagnosis, and Management. Surg Clin North Am. 2019;99:175-84. https://doi.org/10.1016/j.suc.2018.11.002.

[14] Maroni L, Ninfole E, Pinto C, Benedetti A, Marzioni M. Gut-Liver Axis and Inflammasome Activation in Cholangiocyte Pathophysiology. Cells. 2020;9:736. https://doi.org/10.3390/cells9030736.

[15] Khov N, Sharma A, Riley TR. Bedside ultrasound in the diagnosis of nonalcoholic fatty liver disease. World J Gastroenterol. 2014;20:6821-5. https://doi.org/10.3748/wjg.v20.i22.6821.

[16] Charlson ME, Pompei P, Ales KL, MacKenzie CR. A new method of classifying prognostic comorbidity in longitudinal studies: development and validation. J Chronic Dis. 1987;40:373-83. https://doi.org/10.1016/0021-9681(87)90171-8.

[17] Kiriyama S, Takada T, Strasberg SM, Solomkin JS, Mayumi T, Pitt HA, et al. New diagnostic criteria and severity assessment of acute cholangitis in revised Tokyo Guidelines. J Hepatobiliary Pancreat Sci. 2012;19:548-56. https://doi.org/10.1007/s00534-012-0537-3.

[18] Wada K, Takada T, Kawarada Y, Nimura Y, Miura F, Yoshida M, et al. Diagnostic criteria and severity assessment of acute cholangitis: Tokyo Guidelines. J Hepatobiliary Pancreat Surg. 2007;14:52-8. https://doi.org/10.1007/s00534-006-1156-7.

[19] Sbeit W, Greener T, Kadah A, Mari A, Goldin E, Khoury T, et al. Pancreatobiliary manifestations of nonalcoholic fatty liver disease: a retrospective case-control multicenter study. Eur J Gastroenterol Hepatol. 2020. https://doi.org/10.1097/MEG.0000000000001780.

[20] Nseir W, Artul S, Nasrallah N, Mahamid M. The association between primary bacteremia of presumed gastrointestinal origin and nonalcoholic fatty liver disease. Dig Liver Dis. 2016;48:343-4. https://doi.org/10.1016/j.dld.2015.10.004.

[21] Nseir WB, Mograbi JM, Amara AE, Abu Elheja OH, Mahamid MN. Non-alcoholic fatty liver disease and 30-day all-cause mortality in adult patients with community-acquired pneumonia. QJM. 2019;112:95-9. https://doi.org/10.1093/qjmed/hcy227.

[22] Nseir W, Amara A, Farah R, Ahmad HS, Mograbi J, Mahamid M. Non-alcoholic Fatty Liver Disease is Associated with Recurrent Urinary Tract Infection in Premenopausal Women Independent of Metabolic Syndrome. Isr Med Assoc J. 2019;21:386-9.

[23] Nseir WB, Hussein SHH, Farah R, Mahamid MN, Khatib HH, Mograbi JM, et al. Nonalcoholic fatty liver disease as a risk factor for Clostridium difficile-associated diarrhea. QJM. 2020;113:320-3. https://doi.org/10.1093/qjmed/hcz283. 
[24] Tilg H, Burcelin R, Tremaroli V. Liver tissue microbiome in NAFLD: next step in understanding the gut liver axis? Gut. 2020;69:1373-4. https://doi.org/10.1136/gutjnl-2019-320490.

[25] Kim CH, Younossi ZM. Nonalcoholic fatty liver disease: a manifestation of the metabolic syndrome. Cleve Clin J Med. 2008;75:721-8. https://doi.org/10.3949/ccjm.75.10.721.

[26] Hotamisligil GS. Inflammation and metabolic disorders. Nature. 2006;444:860-7. https://doi.org/10.1038/nature05485.

[27] Foroughi M, Maghsoudi Z, Khayyatzadeh S, Ghiasvand R, Askari G, Iraj B. Relationship between non-alcoholic fatty liver disease and inflammation in patients with non-alcoholic fatty liver. Adv Biomed Res. 2016;5:28. https://doi.org/10.4103/2277-9175.176368.

[28] Goldwasser P, Feldman J. Association of serum albumin and mortality risk. J Clin Epidemiol. 1997;50:693-703. https://doi.org/10.1016/s0895-4356(97)00015-2.

[29] Koenig G, Seneff S. Gamma-Glutamyltransferase: A Predictive Biomarker of Cellular Antioxidant Inadequacy and Disease Risk. Dis Markers. 2015;2015:818570. https://doi.org/10.1155/2015/818570.

[30] Liu Y, Zhong G-C, Tan H-Y, Hao F-B, Hu J-J. Nonalcoholic fatty liver disease and mortality from all causes, cardiovascular disease, and cancer: a meta-analysis. Sci Rep. 2019;9:11124. https://doi.org/10.1038/s41598-019-47687-3.

\section{Figures}

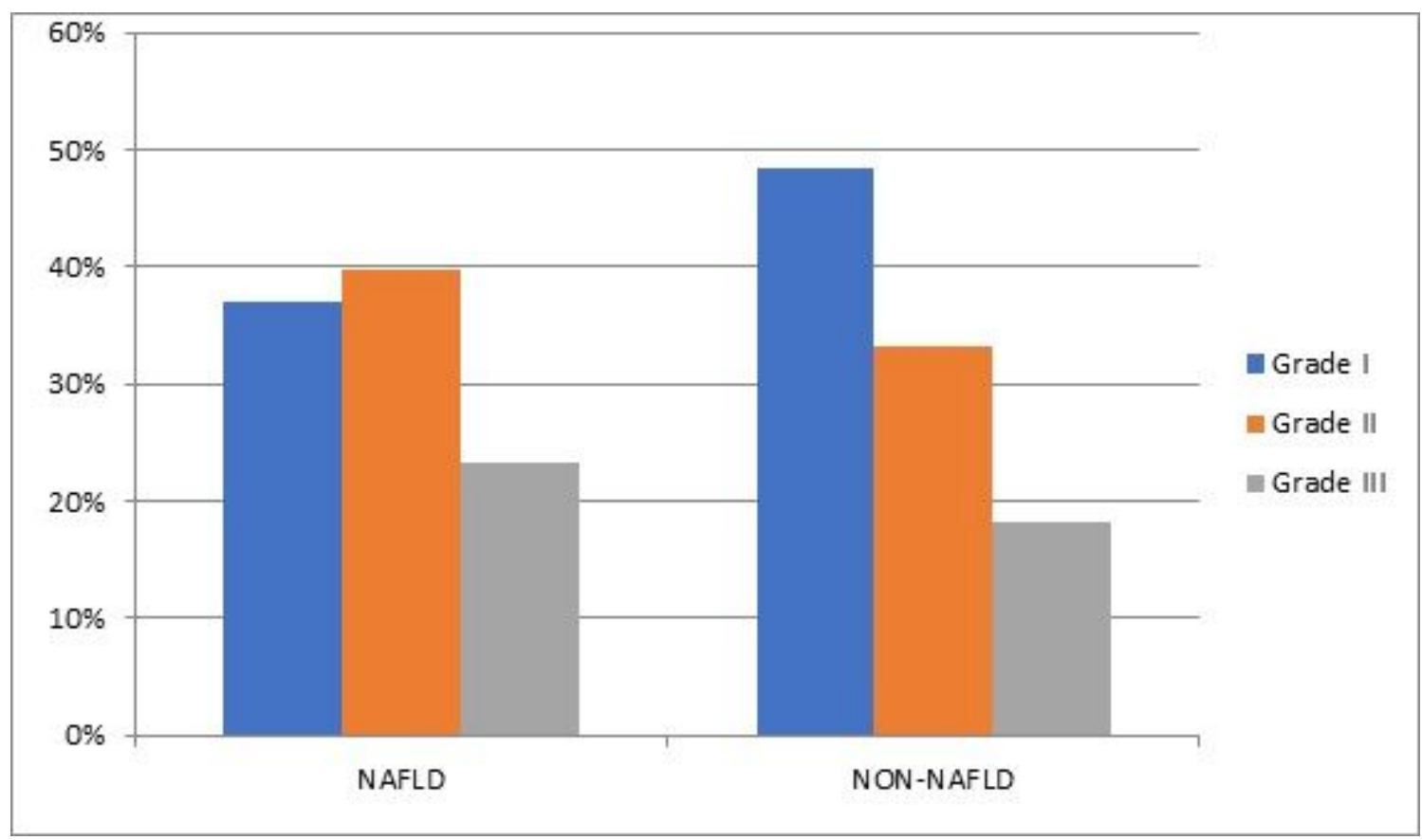

Figure 1 
The association between NAFLD and the severity of cholangitis; measured by Tokyo Score. Abbreviations - NAFLD - non-alcoholic fatty liver disease. 